\title{
PROCESO ADMINISTRATIVO: FACTOR DETERMINANTE EN EL DESARROLLO ORGANIZACIONAL DE LAS MIPYMES
}

\section{ADMINISTRATIVE PROCESS: DETERMINING FACTOR IN THE ORGANIZATIONAL DEVELOPMENT OF MSMES}

\author{
Gary Orlindo Arturo Vasquez Ponce ${ }^{1}$, Darío Hernán Parrales Pilozo², Verónica Elizabeth \\ Morales Chávez ${ }^{3}$ \\ 1,2,3 - Universidad Estatal del Sur de Manabí, Manabí, Ecuador
}

1. Email: gary.vasquez@unesum.edu.ec ORCID: https://orcid.org/0000-0002-6850-5166

2. Email: parrales-dario6436@unesum.edu.ec ORCID: https://orcid.org/0000-0003-1300-9568

3. Email: morales-veronica1776@unesum.edu.ec ORCID: https://orcid.org/0000-0001-6803-3560

Recibido: 11/05/2021 Aceptado: 23/07/2021

Para Citar: Vasquez Ponce, G. O. A., Parrales Pilozo, D. H., \& Morales Chávez, V. E. (2021). Proceso administrativo: factor determinante en el desarrollo organizacional de las mipymes. Revista Publicando, 8(31), 258-278. https://doi.org/10.51528/ rp.vol8.id2249

\section{Resumen: \\ Hoy en día las MiPymes, aplican la gestión administrativa de manera deficiente, generando una baja competitividad, productividad y déficit económico a las organizaciones. Es importante destacar que este sector presenta un problema latente en el ámbito del desarrollo organizacional, teniendo una desventaja a nivel del entorno laboral, ocasionando un mal funcionamiento en su entorno y por ende arriesgando su sostenibilidad, originando que muchas empresas en menos de 5 años se vean obligadas a cerrar sus actividades económicas. Por consiguiente uno de los factores que limitan el desarrollo organizacional es el bajo nivel de capacitación del talento humano, así como los mandos gerenciales que lideran dentro de la organización, generando un clima laboral insano. La presente investigación tiene como objetivo establecer la importancia del proceso administrativo en el desarrollo organizacional, así como identificar los problemas que tienen las MiPymes en Ecuador por causa de una carente aplicación de los procesos administrativos. Para realizar la investigación utilizamos la metodología de investigación bibliográfica o documental, no experimental, como resultado se pudo establecer que la mayoría de las MiPymes no aplican los procesos administrativos, debido a limitantes como deficiente planificación y débil capacidad de financiamiento, ocasionando que se genere un débil desarrollo organizacional afectando al entorno laboral y por ende a la sostenibilidad organizacional.}

Palabras clave: desarrollo organizacional, entorno laboral, MiPymes, proceso administrativo, productividad, sostenibilidad.

\begin{abstract}
:
Nowadays, MSMEs apply administrative management in a deficient manner, generating low competitiveness, productivity and economic deficit to the organizations. It is important to emphasize that this sector presents a latent problem in the field of organizational development, having a disadvantage at the level of the work environment, causing a bad performance in their environment and therefore risking their sustainability, causing many companies in less than 5 years to be forced to close their economic activities. Consequently, one of the factors that limit organizational development is the low level of training of human talent, as well as the management that leads within the organization, generating an unhealthy work environment. The objective of this research is to establish the importance of the administrative process in organizational development, as well as to identify the problems that MSMEs in Ecuador have due to a lack of application of administrative processes. To conduct the research we used the bibliographic or documentary research methodology, non-experimental, as a result it could be established that most MSMEs do not apply administrative processes, due to limitations such as poor planning and weak financing capacity, causing a weak organizational development affecting the work environment and therefore the organizational sustainability.
\end{abstract}

Keywords: organizational development, work environment, MSMEs, administrative process, productivity, sustainability. 


\section{INTRODUCCIÓN}

$\mathrm{E}$ $\mathrm{n}$ la actualidad los procesos administrativos han adquirido un gran valor, siendo estos utilizados como una de las herramientas clave para alcanzar el desarrollo organizacional y este para mejorar la competitividad y productividad en las organizaciones. Cabe destacar que en las MiPymes de Ecuador está latente una gran problemática originada por la deficiencia en la aplicación de los procesos administrativos, causando debilidad en el entorno empresarial de este sector de la economía ecuatoriana, provocando baja productividad, poca competitividad e innovación llevando a las empresas a no ser sostenibles en el tiempo.

En este contexto, el objeto de la investigación consiste en identificar qué factores inciden en la deficiente aplicación de los procesos administrativos y organizacionales en las MiPymes ecuatorianas.

Cabe mencionar que de las micro, pequeñas y medianas empresas en Ecuador son pocas las organizaciones que tienen la capacidad de adaptarse a las tendencias fluctuantes del mercado que cada vez es más globalizado, ocasionando una brecha entre productividad, sostenibilidad y competitividad, producto de algunas barreras de entrada y salida existente en los diferentes entornos económicos en los que se desenvuelven las Mipymes ecuatorianas, así como el limitado acceso a recursos económicos, el poco desarrollo eficiente y eficaz de sus actividades, así como la baja cultura de planificación y gestión administrativa financiera, además es importante destacar que el desconocimiento de los jefes o líderes de estas organizaciones los lleva a improvisar o generar mala toma de decisiones en el desarrollo de la actividad empresarial, afectando a las empresas en su sostenibilidad económica y en algunos casos generando capital de trabajo, sólo para sobrevivir en el mercado.

Para Arzube (2017) en su artículo científico La administración y su relación con el desarrollo organizacional, fundamenta que el proceso administrativo es la herramienta que se debe aplicar en las organizaciones para el logro de los objetivos y satisfacer las necesidades lucrativas. Al igual que en otras actividades se debe aplicar el proceso administración, permitiendo realizar las tareas asignadas eficientemente llevándolas a cumplir su propósito.

En la actualidad la gerencia administrativa se presenta ante un constante cambio e innovación por el cual es necesario la aplicación y desarrollo de herramientas capaces de sistematizar e integrar los procesos administrativos para mostrar así una visualización eficiente de lo que en realidad hacen las empresas. Por tal motivo el proceso administrativo y el desarrollo organizacional, son importantes para que sobrevivan las empresas en el tiempo permitiéndoles ser más competitivas y llevandolas a adaptarse a las nuevas tendencias del mercado (Marlene N. Solís Sierra, 2018).

Sin embargo, Mora et al. (2017) mencionan que los procesos administrativos y financieros son muy importantes para el desarrollo de las empresas, porque sus resultados son la base para las decisiones que toman sus gerentes.

Por otra parte, el desarrollo organizacional es un enfoque del cambio como producto de la intervención planificada, estratégica y sistematizada para fortalecer los recursos, las 
capacidades y las competencias empresariales. En los procesos de desarrollo organizacional se identifican, generan y potencializan la producción en las empresas llevando a impulsar cambios positivos. Además, lleva que muchas empresas tomen decisiones más eficientes permitiendo la resolución de conflictos internos y fortaleciendo las relaciones entre el trabajador y el empleador (Aguilar, 2018).

Además, el conocimiento es indentificado como un recurso fundamental en las organizaciones ya que permite tener un potencial ilimitado para el crecimiento de este gremio, debido a que se provee una ventaja competitiva, sostenible y genera rendimientos crecientes en los estudios de mercado. En este entorno las entidades necesitan aumentar su capacidad para hacer frente a los cambios, necesitan innovar para ser competitivas y sostenibles y para ello se requiere de organizaciones creativas, flexibles, con iniciativa, con visión global y que aporten valor agregado a su competencia para llegar a ser exitosas (Valdés, 2019)

Según Napa (2020), en su artículo científico Modelos Administrativos: Un estudio al Desarrollo Empresarial de las Pymes del sector industrial señala que el ámbito empresarial y la administración han retomado su importancia por su naturaleza, las empresas que aplican de manera correcta los procesos han logrado desarrollar sus actividades de forma eficiente y eficaz esto se ve reflejado en los diferentes objetivos que buscan alcanzar, sin embargo no todas las empresas cumplen a cabalidad con los procesos administrativos, por falta de conocimiento, dudas, temor al fracaso o por no salir de la zona de confort por el contrario, los avances tecnológicos, la globalización y la alta competitividad han impedido desarrollar las micro, pequeñas y medianas empresas.

Los procesos administrativos son un tema de gran escala para la empresa, ya que se trabaja en función de buscar el mayor aprovechamiento de los recursos; teniendo en cuenta que por medio de éstas se integran, se formulan y se aprovechan los recursos, generando mejoría y cambio positivo cuando se lleva a cabo de la mejor manera. Además, es el conjunto de capacidad, conocimientos, actitudes que se obtienen y a la vez adquieren las personas con el transcurso del tiempo, es decir con la experiencia y la responsabilidad para capacitarse y aplicarlo a las distintas áreas de la empresa, para así lograr el mayor objetivo (Orozco, 2015).

De acuerdo con Chiavenato (2015), en su libro Introducción a la teoría general de la Administración el Proceso Administrativo está conformado de la siguiente manera:

Figura 1. Proceso Administrativo

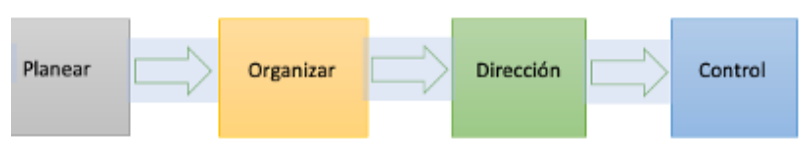

Fuente: Libro Fundamentos de Administración. Autor: Idalberto Chiavenato. 
Tabla 1

Etapas del proceso administrativo .

\begin{tabular}{|c|c|c|}
\hline \multicolumn{3}{|c|}{ PROCESOS ADMINISTRATIVO } \\
\hline ETAPAS & CONCEPTO & AUTOR \\
\hline Planear & $\begin{array}{l}\text { La planeación es la función administrativa que } \\
\text { determina anticipadamente cuáles son los } \\
\text { objetivos que deben alcanzarse y qué debe } \\
\text { hacerse para alcanzarlos. Consiste en tomar } \\
\text { decisiones anticipadas sobre lo que debe } \\
\text { hacerse antes de la ejecución de la acción. } \\
\text { Desde el punto de vista formal, planear, reside } \\
\text { en simular el futuro esperado y establecer } \\
\text { previamente los cursos de acción necesarios y } \\
\text { los medios adecuados para alcanzar los } \\
\text { objetivos. }\end{array}$ & (Chiavenato, 2017) \\
\hline Organizar & $\begin{array}{l}\text { La organización significa el acto de organizar, } \\
\text { estructurar e integrar los recursos y los órganos } \\
\text { involucrados en la ejecución y establecer las } \\
\text { relaciones entre ellos y las atribuciones de cada } \\
\text { uno. }\end{array}$ & (Chiavenato, 2017) \\
\hline Dirección & $\begin{array}{l}\text { La dirección está relacionada con la acción, con } \\
\text { la puesta en marcha y tiene mucho que ver con } \\
\text { las personas. Está directamente relacionada con } \\
\text { la disposición de los recursos humanos de la } \\
\text { empresa. Las personas necesitan ser asignadas a } \\
\text { sus cargos y funciones, ser entrenadas, guiadas } \\
\text { y motivadas para alcanzar los resultados que se } \\
\text { esperan de ellas. La función de dirección se } \\
\text { relaciona directamente con la manera de } \\
\text { orientar la actividad de las personas que } \\
\text { componen la organización para alcanzar el } \\
\text { objetivo o los objetivos. }\end{array}$ & (Chiavenato, 2017) \\
\hline Controlar & $\begin{array}{l}\text { La finalidad del control es asegurar que los } \\
\text { resultados de aquello que se planeó organizó y } \\
\text { dirigió, se ajusten tanto como sea posible a los } \\
\text { objetivos establecidos. La esencia del control } \\
\text { reside en comprobar si la actividad controlada } \\
\text { consigue o no los objetivos o los resultados } \\
\text { esperados. El control es, fundamentalmente, un } \\
\text { proceso que guía la actividad ejecutada hacia un } \\
\text { fin determinado. }\end{array}$ & (Chiavenato, 2017) \\
\hline
\end{tabular}

Fuente: Introducción a la teoría general de la administración: Autor del Libro: Chiavenato. Autores: Darío et al.

Para Moya (2018), las micro, pequeñas y medianas empresas (MiPymes), son de gran importancia en la actividad económica de los países en vías de desarrollo, dada su gran participación en el tejido empresarial, a través de su aporte en la innovación, producción, distribución de bienes y servicios, permitiendo ser generador de empleo en la mayor parte de sectores 
Tabla 2

Histórico y situación deseada para regiones menos competitivas.

\section{CLASIFICACIÓN DE MYPYMES SEGÚN SU TAMAÑO}

\begin{tabular}{|c|c|c|c|}
\hline Microempresa & $\begin{array}{l}\text { Poseen de } 1 \\
\text { hasta } 9 \\
\text { empleados }\end{array}$ & $\begin{array}{l}\text { El valor bruto de las } \\
\text { ventas anuales es } \leq \\
100.000\end{array}$ & $\begin{array}{l}\text { Los montos de } \\
\text { activos son hasta } \\
100.000 \text { dólares }\end{array}$ \\
\hline Pequeña Empresa & $\begin{array}{l}\text { Tienen de } 10 \\
\text { hasta } 49 \\
\text { Trabajadores }\end{array}$ & $\begin{array}{l}\text { El valor bruto de las } \\
\text { ventas anuales es } \\
100.001 \text { hasta } \\
1.000 .000\end{array}$ & $\begin{array}{l}\text { Los Montos de } \\
\text { activos son de } \\
\$ 100.001 \text { hasta } \\
\$ 750.000 \text { dólares }\end{array}$ \\
\hline Mediana Empresa & $\begin{array}{l}\text { Poseen de } 50 \\
\text { hasta } 199 \\
\text { Trabajadores }\end{array}$ & $\begin{array}{l}\text { El valor bruto de las } \\
\text { ventas anuales es } \\
1.000 .001 \text { hasta } \\
5.000 .000\end{array}$ & $\begin{array}{l}\text { Los Montos de } \\
\text { activos son de } \\
\$ 750.001 \text { hasta } \\
\$ 3.999 .999 \text { dólares. }\end{array}$ \\
\hline
\end{tabular}

Fuente: Ámbito financiero. Autores: Dario et al.

de la economía. Además es importante destacar que las MiPymes ecuatorianas se las ha clasificado de acuerdo con el número de empleados y el valor de las ventas anuales (Tabla 2).

\section{MiPymes en Ecuador}

El boletín del Directorio de Empresas y Establecimientos (2020) y el Instituto Nacional de Estadísticas y Censo (INEC) de Ecuador establecen que el total de MiPymes que existen es de 878,454, donde el total de microempresas es de 802.353, el total de pequeñas empresas es de 61.759 y el total de medianas empresas es de 10,110.
Las principales actividades de este sector empresarial son: el comercio, los servicios, las industrias manufactureras, la explotación de minas y canteras. Agricultura, ganadería, silvicultura, pesca y construcción. Sin embargo, el total de unidades económicas es de 882.766. Donde 393.272 unidades económicas se dedican al sector de servicios, 299.231 empresas al comercio, 84.540 negocios corresponden a la agricultura, ganadería, silvicultura y pesca. 74.26 corresponde a la industria manufacturas, 29.633 a la construcción y el total de empresas de explotación de minas y canteras es de 19.824 en Ecuador.

Tabla 3.

Cantidad y porcentaje de las Mypymes.

\begin{tabular}{lll}
\hline SEGÚN TAMAÑO & CANTIDAD & PORCENTAJE \\
\hline Microempresa & 802.353 & $91 \%$ \\
Pequeñas Empresas & 61.759 & $7 \%$ \\
Mediana Empresas “A” & 8.544 & $1 \%$ \\
Mediana Empresas “B” & 5.798 & $1 \%$ \\
TOTAL & 878.454 & $100 \%$ \\
\hline
\end{tabular}

Fuente: Directorio de Empresas y Establecimientos. Autores: Dario Parrales, Veronica Morales. 
Figura 2

El total de número de las micro, pequeñas y medianas empresas que existen en Ecuador del año 2019.

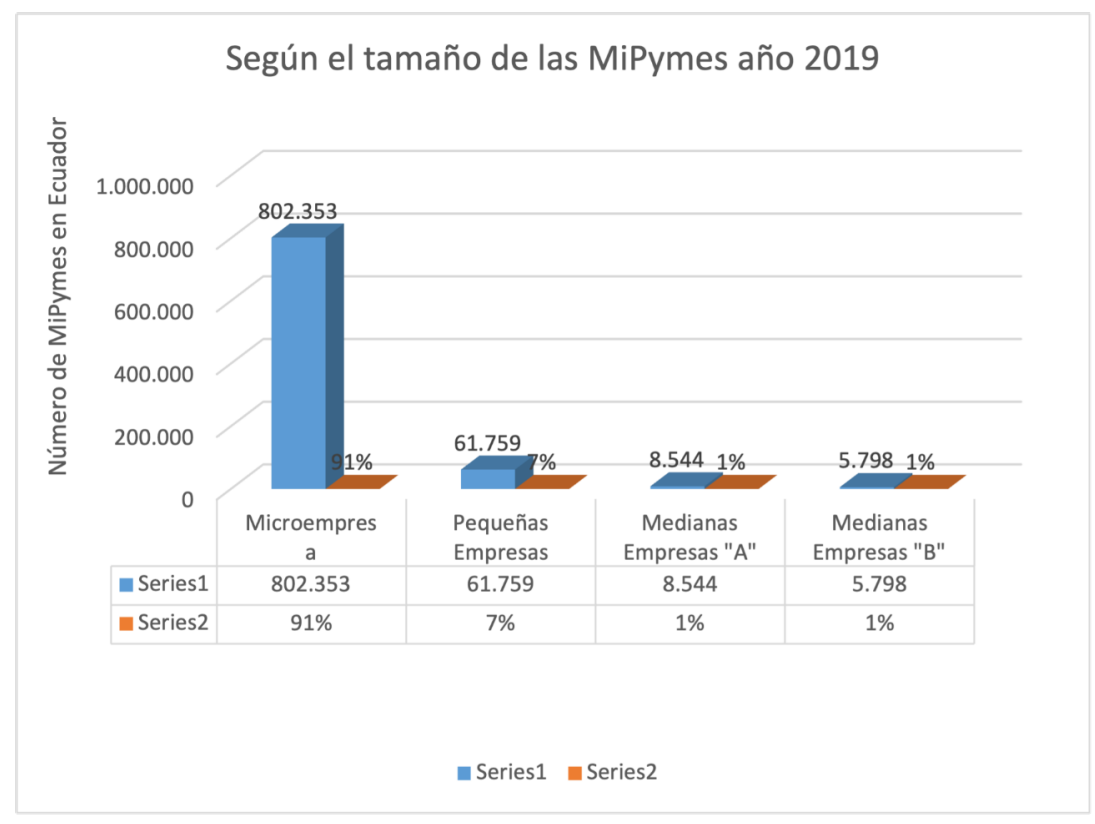

Fuente: Instituto Nacional de Estadística y Censo. Autores: Darío Parrales, Verónica Morales.

La cultura y el conocimiento de una empresas de Ecuador durante los últimos años organización es fundamental para llegar al éxito, es considerando desde el año 2012 hasta el año 2019. importante saber cómo van evolucionando las

Figura 3

Empresas registradas en el SRI y en el IESS.

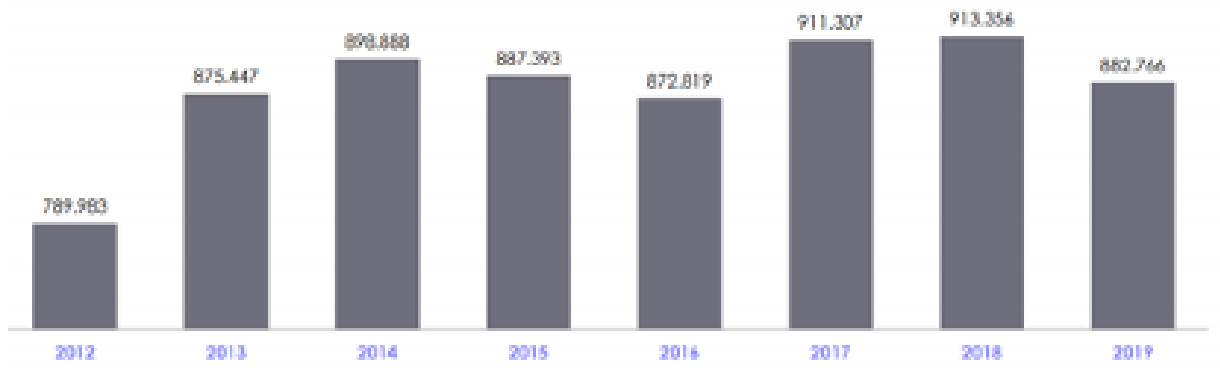

Fuente: Directorio de Empresas y Establecimientos 2019, INEC. 
En esta grafica se consideró las empresas registradas en el SRI y en el IESS durante el período 2012- 2019, estableciendo que en el año 2019 se contaba con un total de 882.766 empresas activas, evidenciándose una disminución de 30.590 MiPymes en contraste con el 2018, es decir, una variación equivalente al $-3.35 \%$. Este escenario es contrario al 2018, en donde se muestra que el número de empresas experimentó un incremento del $0.22 \%$ en comparación con el año 2017.

\section{Los procesos Administrativos $y$ las MiPymes}

La aplicación correcta del proceso administrativo en cualquier tipo de organización es fundamental para el buen funcionamiento de estas debido a que cada etapa se interrelaciona entre sí, formando un proceso integral para que la organización se desarrolle de forma eficiente todos los recursos en cada una de sus fases.

Para Valero (2016), en su artículo científico El proceso administrativo en las MiPymes publicado por la revista Tlamati, México, menciona que la planeación es esencial para cualquier organización porque a través de ésta se toman medidas para reducir los riesgos y aprovechar al máximo las oportunidades que se presenten, así como el tiempo y los recursos en todos los niveles de la empresa.

La organización supone el establecimiento de una estructura intencionada para garantizar la asignación de todas las tareas necesarias para el cumplimiento de las metas. Esta es importante debido a que es un medio a través del cual se establece la mejor manera de lograr los objetivos, evitar la lentitud e ineficiencia de las actividades y reduce o elimina la duplicidad de esfuerzos al delimitar las funciones y responsabilidades ( $p$. 434).

La dirección consiste en influir en los individuos para que contribuyan al cumplimiento de las metas de la organización. Ésta es importante debido a que a través de ella se establece la comunicación necesaria para que la organización funcione, se logren los objetivos evitando así la lentitud e ineficiencia de las actividades y que se reduzca la duplicidad de esfuerzos al delimitar las funciones y responsabilidades (p. 435).

El control es la última etapa del proceso administrativo, se encarga de corregir y medir el desempeño individual y organizacional. La importancia de éste es que se puede aplicar a las cosas y a las personas, reduciendo y ahorrando tiempo al evitar errores (p. 436).

\section{Desarrollo organizacional}

Para Chiavenato (2015), en su libro Introducción a la teoría general de la Administración. Define la organización como la coordinación de diferentes actividades de contribuyentes individuales con la finalidad de efectuar transacciones planeadas con el ambiente. Donde se utiliza la noción tradicional de división del trabajo al referirse a las diferentes actividades. La coordinación se refiere a personas como contribuyentes de las organizaciones, en lugar de que las personas estén totalmente incluidas en las organizaciones. Las contribuciones de cada participante dependen de sus diferencias individuales y del sistema de recompensas y contribuciones de la organización.

El desarrollo organizacional se enfoca 
esencialmente en el lado humano de la institución, de tal manera los valores, las actitudes, las relaciones y el clima organizacional, permiten que se cumplan sus objetivos. Sin embargo, la clave del desarrollo en las organizaciones está en tener en cuenta la creatividad, motivación, compromiso de las personas, fundamentando los altos niveles de competitividad llevandolas a nuevas formas de compromisos, de tal manera se puede decidir y dirigir cómo gestionar las relaciones humanas en las organizaciones de una forma más efectiva. (Pérez, 2016)

Además, la autora Parrales (2017), en su libro Lineamientos Estratégicos, menciona que "el posicionamiento se utiliza para diferenciar el producto y asociarlo con los atributos deseados por el consumidor"(pág. 33). Para ello se requiere tener una idea realista sobre lo que opinan los clientes de lo que ofrece la empresa y también saber lo que se quiere que los clientes meta piensen de nuestra mezcla de marketing y de la de los competidores.

Pérez (2016) en su artículo científico Desarrollo organizacional. Una mirada desde el ámbito académico redacta: "El desarrollo organizacional busca lograr un cambio planeado en las organizaciones asándose por sus necesidades, exigencias o demandas de tal manera la atención se puede concentrar en las modalidades de acción de determinados grupos para así mejorar las relaciones humanas, los factores económicos y de costos, las relaciones entre grupos y el desarrollo de los equipos humanos para una dirección exitosa”.

En este contexto Chiavenato (2015), menciona que la organización es un sistema humano y complejo, con características propias típicas de su cultura y clima organizacional, Ese conjunto de variables debe observarse, analizarse $y$ perfeccionarse continuamente para que resulte en motivación y productividad. Para cambiar la cultura y el clima organizacional, la organización necesita tener capacidad innovadora, permitiendo: la Adaptabilidad, Sentido de identidad, Perspectiva exacta del medio ambiente, integración entre los participantes. Sin embargo, para el desarrollo de estos gremios se debe tomar en cuenta lo siguiente:

- Constante y rápida mutación del ambiente. El mundo moderno se caracteriza por cambios rápidos, constantes y en una progresión explosiva. Donde existen cambios científicos, tecnológicos, económicos, sociales, políticos, etcétera, que influencian el desarrollo y el éxito de las organizaciones.

- Necesidad de continua adaptación. El individuo, la organización y la comunidad son sistemas dinámicos y vivos de adaptación, ajuste y reorganización, como condición básica de supervivencia en un ambiente en continuo cambio.

- Interacción entre individuo y organización. La organización es un sistema social. El DO parte de una filosofía sobre el ser humano dotado de aptitudes para la productividad que pueden permanecer inactivas si el contexto en que vive y trabaja es restrictivo y hostil, impide el crecimiento y la expansión de sus potencialidades.

- El cambio organizacional debe planearse. Es un proceso continuo y complejo para cambiar una empresa, se necesita cambiarla 
$\begin{array}{lllllll}R & \text { E } & \text { V } & \text { I } & S & \text { T } & \text { A }\end{array}$

Public a n d o

I S S N $133900-9304$

toda e involucrar a todos los miembros de la organización en un compromiso conjunto. Se necesita que los líderes dirijan el cambio para que se transmita la actitud positiva al cambio desde arriba hasta abajo. El cambio debe ser responsabilidad personal de todos los encargados de los resultados de la empresa.

- La necesidad de participación y de compromiso. El cambio planeado es una conquista colectiva y no el resultado del esfuerzo de pocas personas. Las resistencias son normales cuando se trata de explorar actitudes, creencias, valores y conductas ya sedimentados y sólidos en las relaciones entre los participantes. El aprendizaje de nuevas conductas debe mejorar la competencia interpersonal (relación humana sin bloqueos y prejuicios) y la adaptabilidad a los cambios.

- La mejora de la eficacia organizacional y del bienestar de la organización depende de la comprensión y de la aplicación de los conocimientos sobre la naturaleza humana. Las ciencias de la conducta permiten localizar y crear el ambiente de trabajo óptimo, en que cada persona pueda dar su mejor contribución $\mathrm{y}$, al mismo tiempo, tener conciencia de su potencial. Las ciencias de la conducta permiten hacer alteraciones y cambios de forma al crear un mínimo de perturbación e interferencias negativas.

- El desarrollo organizacional es una respuesta a los cambios. Es un esfuerzo reduccional complejo, destinado a cambiar actitudes, valores, conducta y estructura de la organización, de tal forma que esa pueda adaptarse a las demandas ambientales, caracterizadas por nuevas tecnologías, nuevos mercados, nuevos problemas y desafíos.

\section{Desarrollo organizacional en las Mipymes}

Lizandro et al., (2016), en su artículo científico El comportamiento organizacional y su importancia para la administración de empresas, menciona que el análisis del comportamiento organizacional, como eje dinamizador de la eficiencia y eficacia de las empresas, constituye hoy en día una abstracción de suma importancia. La clave de un exitoso proceso acertado en el desarrollo de las organizaciones está en la actitud de las personas que participan en ella, de ahí que el comportamiento organizacional sea una herramienta necesaria para beneficio de todo tipo de empresas.

Además, el autor menciona que el resultado de una adecuada gestión organizacional obedecerá en gran medida a cómo se da la motivación, la creatividad, la productividad y el sentido de pertenencia de los miembros de la organización para lograr un mejor uso del capital humano, lo que tributará a generar ventajas competitivas que contribuyan al aumento de los beneficios de la empresa. Los diferentes procesos que inciden en el comportamiento organizacional y que devienen sus diferentes niveles de análisis permiten establecer adecuadas estrategias para comprender y mejorar los retos del cambio empresarial, convirtiéndose así en la llave maestra para alcanzar un liderazgo eficaz.

El desarrollo organizacional está relacionado con el cambio y la capacidad de adaptación de la organización permitiendo que las MiPymes tengan un buen ambiente en el clima laboral. Sin 
embargo, el desarrollo de este gremio no se genera únicamente por los cambios en la estructura organizacional, también se deben enfocar en la cultura organizacional. Es decir, representan las normas informales y no escritas que orientan la conducta de los miembros de la organización día a día y que les dan sentido a sus acciones para la realización de los objetivos organizacionales. Sin embargo, se puede mencionar que cada organización tiene su propia cultura corporativa (Chiavenato, 2015).

De acuerdo con lo anterior expuesto por Chiavenato se puede mencionar que las MiPymes para que tengan un desarrollo en su entorno se deben basar en un buen funcionamiento en el clima laboral, permitiendo evitar conflictos en su entorno llevándolos a tener una mejor comunicación entre jefes o principales $y$ trabajadores. Cabe recalcar que estos factores son de gran importancia para toda organización de tal manera que la utilización de un buen proceso administrativo lleva a trabajar eficazmente en diferentes áreas de la organización, permitiendo mejorar su productividad, competitividad $y$ adaptarse a los nuevos cambios del mercado. Sin embargo, podemos determinar si las organizaciones desean mantenerse, crecer $y$ desarrollarse en un entorno dinámico tienen que plantear estrategias que les permitan alcanzar su desarrollo empresarial.

\section{MÉTODOS Y RESULTADOS}

Para el estudio, se recopiló información en diferentes fuentes bibliográficas como libros, revistas científicas, wikis, páginas web, entre otros. Considerando el orden cronológico de acuerdo con las dos variables de estudio.
El análisis se enfocó en la logística de la investigación como en la revisión sistemática de documentos, revisiones y estudios científicos, permitiendo estudiar el objeto de las variables de la investigación aprovechando el diseño documental y no experimental. La aplicación del método documental, bibliográfico permitió valorar la información más relevante y realizar una revisión sistemática de los estudios realizados por diferentes autores de la comunidad científica concernientes a las variables de estudio como son los procesos administrativos y el desarrollo organizacional, el cual permitió obtener resultados acerca de este tema de estudio, además de determinar si las MiPymes de Ecuador aplican o no los procesos administrativos como un factor relevante para su desarrollo empresarial.

\section{RESULTADOS}

Para la argumentación de los resultados en la presente investigación se consideró diferentes fuentes bibliográficas, lo cual permitió recopilar información en las temáticas acerca de la aplicación de los procesos administrativos y su importancia como desarrollo organizacional.

Sin embargo, para la obtención de resultados se tomó como referencia la investigación realizada en la provincia de Santa Elena y la Provincia de Esmeralda, basándonos en estudios realizados por otros autores que comparten sus investigaciones con la comunidad científica, donde afirman que estos gremios tienen un porcentaje en aplicación y la falta de aplicación de los procesos administrativos como un factor importante para el desarrollo empresarial.

Álvarez (2015) en su artículo científico Aplicación del proceso administrativo en las 


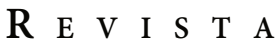 Publi c a n d o \\ I S S N $133900-9304$}

Tabla 4

Estudios sobre aplicación de Procesos administrativos en MiPymes y Desarrollo Organizacional.

\begin{tabular}{llll}
\hline AUTOR & ANO & FINALIDAD & CONCLUSIÓN \\
\hline $\begin{array}{l}\text { Roxana Álvarez } \\
\text { Acosta }\end{array}$ & 2015 & $\begin{array}{l}\text { Determinar si las } \\
\text { MiPymes en Ecuador } \\
\text { aplican los procesos } \\
\text { administrativos para } \\
\text { desarrollo } \\
\text { organizacional. }\end{array}$ & $\begin{array}{l}\text { Las empresas deben trabajar hacia el mejoramiento } \\
\text { continuo, basado en sistema de calidad orientados } \\
\text { al control estadísticos de procesos y que debe } \\
\text { existir una buena motivación y liderazgo de los } \\
\text { administradores para que las empresas sean } \\
\text { competitivas en los mercados locales, regionales, } \\
\text { nacionales e internacionales. }\end{array}$
\end{tabular}

Francisco Mila- 2019 Carvajal

Marco Mauricio 2020 Chávez Haro

$\begin{array}{lll}\text { Lizandro A. 2016 } & \begin{array}{l}\text { Determinar el } \\ \text { Molina Sabando }\end{array} \\ & \text { organizacional y su } \\ & \text { importancia para la } \\ & \text { administración de } \\ \text { empresas. }\end{array}$

Definir la importancia de los procesos administrativos como componentes al desarrollo organizacional de las MiPymes.

Gestión por proceso en las micro, pequeñas y medianas empresas
El promedio es $97.8 \%$ de la MIPYMES no evidencian presencia de la gestión por procesos en sus actividades cotidianas. Esto representa un aspecto crítico en las dimensiones financieras, procesos internos, clientes y de talento humano. Unicamente, el 2.2\% de las MIPYMES aplica gestión por procesos.

La gestión administrativa tiene un papel importante en cualquier empresa y por ende está orientada a lograr los objetivos de la organización, mediante la aplicación de las etapas del proceso administrativo se puede realizar un buen funcionamiento en cualquier área en el entorno empresarial.

El comportamiento organizacional, como eje dinamizador de la eficiencia y eficacia de las empresas, constituye hoy en día una obstrucción de suma importancia. La clave de un exitoso proceso acertado en el desarrollo de las organizaciones está en la actitud de las personas que participan en ella.

El resultado de una apropiada gestión organizacional obedecerá en gran medida de acuerdo con la motivación, la creatividad, la productividad y el sentido de pertenencia de los miembros de la organización para lograr un mejor uso del capital humano, lo que tributará a generar ventajas competitivas que contribuyan al aumento de los beneficios de la empresa.

\section{Moreta Maria 2017} Augusta
Identificar qué factores Existen muchas limitaciones para planificar como limitan la aplicación de son, la disponibilidad de recursos y principalmente los procesos para su desarrollo organizacional

el desconocimiento del nivel directivo sobre

planeación estratégica formal, Falta de formalización del proceso estratégico, manifestaciones culturales, falta de innovación, Software y herramientas inadecuadas, Recursos financieros, Prioridad es la supervivencia y no el crecimiento, influencia familiar en la fijación de la estrategia, falta de formalización del proceso estratégico, objetivos sobrestimados, y resolución de problemas del día a día. 


\begin{tabular}{lll}
\hline José Nivela Icaza 2017 & $\begin{array}{l}\text { Gestión administrativa } \\
\text { de las PYMES y su } \\
\text { incidencia en la } \\
\text { organización del } \\
\text { trabajo }\end{array}$ & $\begin{array}{l}\text { Las pequeñas y medianas empresas juegan el papel } \\
\text { más relevante en el desarrollo local de la economía } \\
\text { y en la integración del mercado de trabajo, por tal } \\
\text { motivo es importante que los empresarios dominen } \\
\text { los instrumentos necesarios para su dirección y } \\
\text { gestión, con la meta de hacer frente al panorama } \\
\text { actual, caracterizado por la alta competitividad }\end{array}$ \\
& & \\
\end{tabular}

empresas de la provincia de Santa Elena, menciona como resultado que en la planeación al momento de revisar los aspectos normativos se encuentra con grandes deficiencias por parte de las empresas, en especial el hecho de no contar con manuales debidamente actualizados. La no publicación de los organigramas en lugares visibles para todos los trabajadores, ocasionan la falta de conocimiento de cómo está constituida la empresa, siendo una desventaja competitiva en los mercados actuales.

Es por ende que los informes que se realizan no están aplicando técnicas de control estadístico de procesos y mejoramiento continuo, causando una desorganización administrativa reflejada en la mala planificación del entrenamiento del personal, enfocadas en técnicas de calidad total, el no reconocimiento de su trabajo mediante recompensas y una buena remuneración, llevan a que el trabajador no ponga mucho empeño laboral, afectando la productividad de las empresas.

Lo anteriormente fundamentado por Álvarez (2015), afirma que si las MiPymes no consideran los procesos administrativos como un factor relevante para su desarrollo, ocasionando a estos gremios en no alcanzar estándares de competitividad y calidad, indicando que las acciones administrativas son de vital importancia para optimizar procesos y aumentar la productividad, como una respuesta rápida y eficaz sobre la mejora continua de los procesos administrativos, contribuyendo al desarrollo organizacional empresarial.

En este contexto Francisico et al. (2019), en su artículo científico Gestión por Procesos en las Micro, Pequeñas y Medianas empresas comerciales de la ciudad Esmeraldas de Ecuador. Indica que el $\mathbf{9 7 . 8 \%}$ de la MiPymes no evidencia presencia de la gestión por procesos en sus actividades cotidianas. Esto representa un aspecto crítico en las dimensiones financieras, procesos internos, clientes y de talento humano, que influyen significativamente en la retención y satisfacción de clientes, puesto que no concurren acciones sistémicas que vinculen todas las dimensiones. Únicamente, el 2.2\% de las MiPymes aplica gestión por procesos.

El proceso administrativo debe ser visto en relación con el entorno y la estrategia, lo cual implica que para estudiarlo se debe partir del diagnóstico situacional de una empresa, de su industria $y$, en relación con el territorio, conocer las oportunidades y amenazas que pueden presentarse para la organización.

En un mundo globalizado, no sólo compiten las empresas sino también las regiones, que tratan de atraerlas para generar empleo y crecimiento, lo cual introduce el tema de la competitividad 
regional como influyente en las estrategias y en los procesos de las empresas.

Es importante destacar que la gestión administrativa tiene un papel preponderante en cualquier empresa ya que esta permite la consecución de los objetivos de la organización mediante la aplicación de las etapas inherentes al proceso administrativo como la planificación, organización, dirección y control. Además permite a una empresa contribuir a elevar los resultados económicos, ayudando no solo a la economía interna de la unidad productiva, sino también con el desarrollo de la localidad y del país (Chávez, 2020).

Para determinar qué factores limitan la aplicación de los procesos administrativos en las MiPymes del Ecuador se ha considerado las investigaciones realizadas por diversos autores ecuatorianos.

Moreta (2017) quien en su artículo científico Planeación estratégica en Pymes: limitaciones, objetivos y estrategias, menciona que hay muchas limitaciones para planificar como son, la disponibilidad de recursos y principalmente el desconocimiento del nivel directivo sobre planeación estratégica formal. Sin embargo, los ambientes complejos e inciertos afectan el desempeño de estas empresas y en muchas ocasiones las llevan a la quiebra.

Además la deficiente planeación estratégica es una de las razones por las cuales este sector empresarial no sobrevive a largo plazo, cabe mencionar que la autora Moreta (2017) establece que factores como la poca formalización de los procesos estratégicos, las manifestaciones culturales, la adaptación de planes de corporaciones grandes, estrategias mal fundamentadas, la no inclusión del recurso humano como parte de la estrategia de la organización, considerando que la planeación es de absoluta responsabilidad de la gerencia, así como los softwares y herramientas inadecuadas, la predominante planeación con un horizonte de corto plazo, la influencia familiar en la fijación de la estrategia, el poco interés en innovación, recursos financieros escasos o limitados por la mala planificación y gestión, ocasiona que en las MiPymes sólo se priorice la supervivencia y no el crecimiento, generando una economía incierta, objetivos sobrestimados y la deficiente resolución de problemas del día a día.

Las limitaciones que se presentan en las pymes, provocan no tener una buena gestión empresarial. Pero al mismo tiempo existen soluciones efectivas para ir superando todas estas barreras y los beneficios que estos traen.

Cabe destacar que Nivela et al. (2017) mencionan que las pequeñas y medianas empresas juegan el papel más relevante en el desarrollo local de la economía y en la integración del mercado de trabajo, por tal motivo es importante que los empresarios dominen los instrumentos necesarios para su dirección y gestión, con la meta de hacer frente al panorama actual, caracterizado por la alta competitividad. Con la ayuda de las Pymes se puede impulsar el desarrollo de una comunidad, porque al mejorar la calidad de vida de su fuerza laboral, estos invierten en el progreso individual y familiar.

Pero el desconocimiento de los procesos administrativos como herramienta estratégica por parte de los principales o administradores 
lleva a las organizaciones a tomar malas decisiones, llevando a la empresa a ser deficiente en la productividad y en el desempeño laboral, ocasionando que las mismas dentro del mercado en el que se desenvuelven no sean sostenibles. Por otra parte la poca capacitación del empleado y empleador lleva a tener un clima desagradable en las empresas.

Todas las limitaciones que tienen las MiPymes de Ecuador en los procesos administrativos llevan a la poca productividad, afectando a su entorno laboral y a su competitividad, lo cual no les permite adaptarse a los cambios fluctuantes del mercado y se tornan entes económicos con un debilitado desarrollo organizacional.

Es así como Lizandro (2016), en su artículo científico El comportamiento organizacional y su importancia para la administración de empresas, argumenta que el comportamiento organizacional, como eje dinamizador de la eficiencia y eficacia de las empresas, constituye hoy en día una arista de suma importancia. La clave de un exitoso proceso acertado en el desarrollo de las organizaciones está en la actitud de las personas que participan en ella, de ahí que el comportamiento organizacional sea una herramienta necesaria, para beneficio de todo tipo de empresas. El resultado de una apropiada gestión organizacional obedecerá en gran medida de acuerdo con la motivación, la creatividad, la productividad y el sentido de pertenencia de los miembros de la organización para lograr un mejor uso del capital humano, lo que tributará a generar ventajas competitivas que contribuyan al aumento de los beneficios de la empresa. Sin embargo, el cambio organizacional es la capacidad de adaptarse a las organizaciones en diferentes transformaciones que sufre el medio ambiente interno o externo, mediante el aprendizaje.

Para la administración de empresas resulta esencial el manejo adecuado de los elementos esenciales del comportamiento organizacional como vía para favorecer el ambiente social y cultural de las empresas, incidiendo en que los empleados cumplan con eficiencia las labores propias de cada una de sus áreas. El logro de un efectivo comportamiento organizacional permitirá que la empresa esté en mejores condiciones de enfrentar los retos de este entorno disruptivo de la economía globalizada.

Además, Nivela (2017) en su artículo científico Gestión administrativa de las Pymes y su incidencia en la organización del trabajo afirma que las Pymes enfrentan hoy el desafío de la internacionalización como oportunidad de crecimiento. Por la deficiente gestión administrativa, saturación, exceso de competencia, organización en el trabajo, reducción de costos, importaciones, entre otros, el mercado obliga a considerar otras opciones para la comercialización de sus productos. Este sector empresarial juega un papel relevante en el desarrollo local de la economía y en la integración del mercado de trabajo, por tal motivo es importante que los empresarios dominen los instrumentos necesarios para su dirección y gestión, con la meta de hacer frente al panorama actual, caracterizado por la alta competitividad.

El principal reto al que se enfrentan las MiPymes para lograr el éxito y generar empleos, es la generación de una capacidad empresarial que les permita operar en forma eficiente y eficaz, para poder tomar decisiones en forma ágil cuando las oportunidades y amenazas del entorno y las 
fortalezas y debilidades internas así lo ameriten.

Por su parte Bonilla (2016) en su investigación menciona que el nivel de liderazgo, motivación, reciprocidad y participación en los trabajadores no es satisfactorio destacando esta problemática en áreas como la administración, almacén y bodega, debido a la poca comunicación entre mandos jerárquicos, medios y operativos, así como la forma de negociación, la cual es inadecuada, por tal razón la esencia de un buen clima laboral está determinada por las oportunidades que se crean para los trabajadores y le corresponde a la empresa propiciar que estas oportunidades se den.

Sin embargo, una propuesta de cambios para mejorar el clima laboral es optimizar la comunicación entre la alta dirección y los trabajadores de línea y hacer participe a los empleados en la toma de decisiones, fomentando confianza en los colaboradores en todas sus líneas. Esto incidirá directamente en la producción de la empresa y el bienestar de todos quienes conforman la institución para el desarrollo empresarial.

Las PYMES de Ecuador, están conformadas por grupo de individuos que se asocian para crear un emprendimiento, de ahí la importancia de que quien esté al frente de este grupo de emprendedores, sea la persona que sepa guiarlos y que tenga las cualidades y destrezas de un verdadero líder, superando las adversidades que se les pueda presentar y aprovechando las oportunidades de los mercados globalizados. Es importante acotar que en muchos de estos emprendimientos quién asume el papel de administrador, no cuenta con habilidades de liderazgo, de ahí la necesidad de que en esta fase se cumpla con una etapa de formación o capacitación en liderazgo para quien esté al frente del equipo de trabajo.

Como se puede apreciar el desarrollo organizacional constituye uno de los factores primordiales a mejorar continuamente dentro de la administración, ya que este permite estructurar un ambiente laboral inclusivo eficiente y eficaz, generando una productividad de calidad de capital humano dentro de la organización, ya que este es uno de los elementos primordiales para garantizar el éxito y sostenibilidad de las empresas en la economía global donde se desarrolla cada una de ellas.

\section{DISCUSIÓN}

Para el desarrollo de la discusión se consideró los trabajos investigativos y bibliográficos expuestos por autores de los diferentes países a nivel de Latinoamérica donde se manejan las Pymes y MiPymes, para lo cual se realizó un contraste considerando estos países extranjeros, la situación y el entorno de las MiPymes en Ecuador, lo cual va a permitir generar una opinión más clara de cómo se están trabajando a nivel de los procesos administrativos y desarrollo organizacional, reflejando tener un mejor panorama en el desarrollo administrativo ecuatoriano a nivel empresarial.

Para García (2014) en su investigación La Gestión por Procesos en las Pymes de Barranquilla: Factor Diferenciador de la Competitividad Organizacional redacta que los aspectos que deben tener los Pymes es considerar los procesos administrativos como un factor importante para su desarrollo empresarial, de tal manera les permitirá controlar todos los recursos 
que tienen, reflejando un buen clima laboral, el mejoramiento en el empeño laboral para esto la empresa también debe considerar la innovación, la capacitación por parte de los empleados y el análisis, esto permitirá saber cuales son las fortalezas y las debilidades que tiene la empresa para luego aplicar estrategia que le permita el desarrollo organizacional.

En los últimos años, el análisis y el diseño administrativo se han enriquecido al involucrar para su comprensión elementos de sistemas y de procesos que permiten mirar el todo y las partes de la organización, la reflexión dentro y fuera de lo general y de la especialización, de la integración interna y de la adaptación externa de la eficiencia y de la eficacia (Sangrona, 2019).

Además, el autor menciona que en la administración, la información es un elemento más de la infraestructura de gestión; sin embargo, la tecnología juega un papel importante, no sólo como herramienta de puesta en marcha de partes del sistema de información, sino por las oportunidades que en sí misma ofrece a la organización.

En estos resultados obtenidos por los países Colombia y Venezuela se puede evidenciar que todas las organizaciones deben considerar los procesos administrativos como dependientes, armónicos, coherentes, equilibrados $y$ complementarios entre sí, que garanticen logros, mejoramientos y cambios organizacionales de tal manera estas actitudes dependen del éxito de las empresas llevándolas a tener un buen crecimiento. De tal manera las MiPymes a nivel de Latinoamérica representan un gran porcentaje en la contribución de generar empleo, proporcionando ingresos la erradicación de la pobreza en la sociedad y fortaleciendo las actividades productivas en la economía local de cada país.

Es por ende que las MiPymes de Ecuador, deben aplicar los procesos administrativos como un factor importante para su desarrollo, ya que en este sector se caracteriza por la poca implementación en la innovación, las estrategias o el cambio en su estructura administrativa, lo cual genera graves problemas a largo plazo para evitar esto, las empresas deben tener un mejor control en toda las áreas o funciones, llevando a tener un buen clima laboral y el mejoramiento de la productividad para jugar un buen papel en la competencia y que estos gremios se adapten en el mercado.

La mala aplicación de los procesos administrativos no permite a las empresas su sostenibilidad a largo plazo, ocasionando tener poca productividad, llevando a tener desventaja en la competencia y la falta de innovación lo cual provoca que las micro, pequeñas y medianas empresas de Ecuador se vean obligadas a cerrar.

La situación de las pymes en Ecuador es poco similar a las empresas de América Latina ya que en ellas se observa la importancia de estas en la economía como grandes empresas generadoras de empleo y productividad económica.

Las limitaciones que llevan a las empresas al declive son porque no analizan el factor interno que poseen estos gremios y el factor externo como es la competitividad y las demandas del mercado, es por esto, que el precio de materias primas, la responsabilidad social y corporativa, el nivel adquisitivo de la población, la ética profesional, la 
estabilidad política, uso, eficiencia energética y utilización de energías renovables, la innovación empresarial, certificación ISO y la ineficiencia del uso de los procesos administrativos, constituyen problemas que enfrentan hoy en día las empresas a nivel global.

Por otra parte, Fonseca (2020) menciona que el poco acceso a financiamiento de las entidades financieras, lleva a las MiPymes a no contar con un crédito para incrementar su capital e incidir a la hora de innovar ya que por falta de recursos económicos lleva a estos gremios a no aprovechar los equipos tecnológicos que existen actualmente para mejorar la productividad y el empeño laboral.

Cabe destacar que de acuerdo con Fonseca las principales limitaciones de las PyMES están en los costes derivados de la información lo que puede causar graves perjuicios, al igual que la falta de financiación; por otro lado, el desconocimiento de las normas nacionales e internacionales trae como consecuencia pérdidas en su exportación o morosidad en el servicio que brindan; la ausencia de alfabetización tecnológica de los empresarios afecta la inclusión en el mercado.

Las PyMES en Paraguay representan un alto por ciento de la industria, el comercio y los servicios, así como en la cantidad de trabajadores; sin embargo, se encuentran por debajo en los ingresos y las remuneraciones pagadas y una de las causas es la carencia de recursos para la internacionalización.

Tomando en consideración de otras investigaciones hechas en México y Paraguay se puede determinar que las limitaciones dentro de las organizaciones afectan gravemente en su entorno, la falta de financiamiento lleva a que todas las empresas no innoven y en general, permite generar poca productividad, llevando a ser poco competitivo, de tal manera la falta de capacitación conlleva a la mala toma de decisiones; es por tal motivo que el personal y el empleador deben estar siempre capacitados permitiendo tener el perfeccionamiento de las habilidades específicas y la inducción y adecuación de las actitudes de las personas, mejorando su rendimiento, favoreciendo a la empresa. Es por esto, por lo que las MiPymes de Ecuador deben considerar las limitaciones que existen a nivel global, para luego utilizar estrategias que le generen sostenibilidad y desarrollo en su entorno empresarial.

Para el desarrollo organizacional la globalización es un proceso económico que abarca a sectores productivos y a regiones del mundo conforme a un determinado patrón de producción y financiamiento. Para esto se tomó como muestra el país México. En este contexto Correa (2013), menciona que el desarrollo económico regional puede sustentarse en una estrategia productiva enfocada no sólo en las ventajas competitivas derivadas de los precios, sino esencialmente de las ventajas asociadas con la diferenciación de los productos y la ubicación de segmentos.

Sin embargo, Porter (2015), fundamenta que las pymes siendo un modelo empresarial de mucha importancia en Colombia y con un impacto tan interesante, se hace recomendable la motivación para aplicar el sistema de gestión por procesos a promover un aporte mejor a la economía local, nacional y mundial; de esta manera se pueden alcanzar niveles de gestión diferenciadores, que no sólo promuevan la competitividad como se ha expresado. 
Asimismo Meza et al. (2019), mencionan que "el desarrollo organizacional se valora como una herramienta administrativa para incrementar productividad, reducir riesgos $y$ ambigüedades en los procesos como también para generar modificaciones en la organización mediante diagnósticos de mejora continua” (pág. 1). Además, los autores hacen referencia a que el clima laboral repercute directamente en la eficiencia y eficacia de las organizaciones y en el desempeño del personal. Un diagnóstico de clima organizacional podría implementar cambios deseados dentro de una organización basados en tendencias de desarrollo, proyecciones $y$ planificaciones que ayuden al desarrollo y bienestar de esta. Por lo tanto, el diagnóstico es una etapa necesaria de cualquier intervención en las organizaciones y sirve como instrumento adecuado para el conocimiento de la organización y para la planificación racional.

Las pymes son organizaciones que se adaptan fácilmente a los cambios generados por la competencia, para ello deben alcanzar un crecimiento económico, cultural, liderazgo, gestión del conocimiento e innovación llevándolos a tener un desarrollo empresarial.

Con los resultados obtenidos se ha tomado como referencia países como México y Colombia permitiendo discutir que las MiPymes actualmente enfrentan muchas barreras a nivel nacional, por ende, en Ecuador estas organizaciones no desarrollan su entorno empresarial, por las limitaciones que enfrentan actualmente. Sin embargo, la pandemia Covid-19 ha afectado gravemente a muchas empresas de Ecuador, ocasionando poca productividad y esto es un factor que no permiten desarrollar a largo plazo. Es importante considerar que estos gremios necesitan de aplicar muchas estrategias, las cuales ayuden a tener una mejor organización empresarial, teniendo en cuenta que un desarrollo organizacional es uno de los elementos más importantes para tener un buen ambiente laboral y así las empresas sigan en camino hacia el éxito.

Para que las MiPymes de Ecuador desarrollen deben enfocarse en utilizar los procesos administrativos como un factor clave para su desarrollo, permitiendo controlar sus recursos y aplicando estrategias que le lleven a ser cada día mejores. Sin embargo, la falta de liderazgo abunda en las empresas, por falta de conocimientos, la falta de innovación lleva a la empresa a no durar mucho tiempo en el mercado; la buena comunicación y el trabajo en equipo para una empresa es vital, por tal movido, se debería poner en práctica a diario en su entorno laboral con la finalidad de realizar todas las tareas asignadas con eficacia y eficiencia. Es por esto por lo que el diagnóstico permitirá saber cómo está habitualmente organizada la empresa y cuáles son las falencias que llevan a estos gremios a no tener limitaciones para su crecimiento empresarial.

\section{CONCLUSIONES}

Se concluye que las MiPymes en Ecuador en su mayoría no aplican una buena gestión administrativa, teniendo un déficit de conocimiento acerca de los procesos administrativos, permitiendo llevar a cabo poca competitividad, productividad y escasos recursos materiales y económicos de tal manera que el desarrollo de estos gremios es muy difícil por esto en su mayoría las MiPymes mueren cada 5 años.

Las limitantes en las Pymes o MiPymes 
$\begin{array}{lllllll}R & \text { E } & \text { V } & \text { I } & S & T & A\end{array}$

Public a n d o

I S S N $113900-9304$

generan un desarrollo organizacional deficiente por la falta de conocimiento del empresario, tanto en el área administrativa como financiera, generando en estas organizaciones empresariales un mal entorno laboral, una limitada implementación de tecnología e innovación en sus procesos productivos y en sus servicios teniendo una participación poco destacable en el mercado y conllevando a estas organizaciones a no tener un posicionamiento destacado en el mismo, por no ser competitivos.

El estudio del desarrollo organizacional es un reto importante para la administración de las MiPymes ya que este permite conocer que tipos de modelos de gestión de recursos humanos se puede aplicar para contribuir al incremento de la eficiencia y eficacia en un clima laboral favorable, tomando como gran importancia la tecnología de gestión permitiéndoles prestar sus servicios a los pequeños grupos o equipo de trabajos. El punto central del clima laboral de las organizaciones es lograr un adecuado comportamiento organizacional llevando a las empresas a tener mejor productividad y ser más competitivas. 


\section{$\begin{array}{lllllll}\text { R } & \text { E } & \text { V } & \text { I } & S & T & A\end{array}$}

Public a n d o

I S S N $13390-9304$

\section{REFERENCIAS}

Aguilar, M. G. (2018). El desarrollo organizacional como generador de un cambio significativo en las organizaciones de la sociedad civil. Extremeña de Ciencias Sociales, 87-109.

Álvarez, J. C. (2015). Aplicación del proceso administrativo en las empresas de la provincia de Santa. Ciencias Multidisciplinarias, 216-226.

Arzube, Z. R. (2017). La administración y su relación con el desarrollo organizacional. CE Contribuciones a la Economía.

Carrillo, L. A. (2014). Necesidades de capacitación de las pymes del Cantón de Bagaces. Revista Reflexiones.

Chávez Haro, Á. G. (2020). La gestión administrativa desde los procesos de las empresas agropecuarias en Ecuador. FIPCAEC, 16-29. https://doi.org/10.23857/fipcaec.v5i18.198

Chiavenato, I. (2015). Introducción a la teoría general de la Administración (Sexta ed.). Cuajimalpa, México, D. F., México: McGraw-Hill.

Chiavenato, I. (2017). Introducción a la teória general de la Administración (7 ed.). (N. I. López, Ed.) méxico, México: McGraw-Hill.

Correa, J. E. (2013). Influencia del capital humano para la competitividad de las pymes en el sector manufacturero de Celaya, Guanajuato. México : Universidad de Celaya.

Directorio de Empresas y Establecimientos. (Octubre de 2020). ecuadorencifras.gob.ec. Obtenido de ecuadorencifras.gob.ec: https:// www.ecuadorencifras.gob.ec/documentos/ web-inec/Estadisticas_Economicas/
Directoriotmpresas/ Directorio_Empresas_2019/ Principales_Resultados_DIEE_2019.pdf

Fonseca, V. C. (2020). Las pequeñas y medianas empresas en Paraguay. Limitaciones para su internacionalización. Revista Internacional de Investigación en Ciencias Sociales.

García. (2014). La Gestión por Procesos en las Pymes de Barranquilla: Factor Diferenciador de la Competitividad Organizacional. Scielo.

García Saltos, F. J. (2016). El proceso adminstrativo en las MiPymes del cantón Machala, provincia del oro. Observatorio de la Economía, 1- 11.

Meza De los Cobos, J. M. (2019). Intervención en el clima laboral para el desarrollo organizacional de una empresa de servicios logísticos. Unisimon, 9-32.

Mila-Carvajal, F., Reyes-Ordoñez, B., Dueñas-Mendoza, A., \& Armas-Arias, M. (2019). Gestión por Procesos en las Micro, Pequeñas y Medianas Empresas comerciales de la ciudad Esmeraldas, Ecuador. Veritas \& Research, 1(2), 140-149.

Molina-Sabando, Í. B. (2016). El comportamiento organizacional y su importancia para la administración de empresas. Domicio de la Ciencia, 2, 498-510.

Molina-Sabando, M. Í.-V.-C. (Octubre de 2016). El comportamiento organizacional y su importancia para la administración de empresas. Dominio de las Ciencias, 498 - 510.

Mora-Aristega, M. R. (2017). Aseguramiento de los procesos administrativos y financieros. Ciencias Administrativas, 3- 15. 
Moreta, M. A. (2017). Planeación estratégica en PYMES: limitaciones, objetivos y estrategias. revistasutc, 1-179.

Moya, D. P. (07 de 12 de 2018). Recuperado el 13 de 02 de 2021, de Gestionar Fácil: https:// www.gestionar-facil.com/mipymes/

Napa, P. B. (2020). Modelos administrativos: un estudio al desarrollo empresarial de las pymes del sector industrial. unesumciencia, 1-14.

Orozco, S. E. (2015). Proceso administrativo y gestión empresarial en Coproabas, Jinotega. Maestría en Gerencia Empresarial (págs. 1-188). Matagalpa: Universidad Nacional Autónoma de Nicaragua, Managua.

Parrales, M. E. (2017). Lineamientos Estratégicos. Jipijapa: Mawil Publicaciones de Ecuador.

Pérez, A. M. (2016). Desarrollo organizacional. Una mirada desde el ámbito académico. Elsevier, páginas 3-8. Recuperado de https:// www.elsevier.es/es-revista-educacionmedica-71-articulo-desarrolloorganizacional-una-mirada-desde$\underline{S} 1575181315000212$

Porter. (2015). La gestión por procesos en las Pymes de Barranquilla: Factor Diferenciador de la Competitividad Organizacional. SCIELO.

Sangrona, O. G. (13 de 02 de 2019). gestionpolis.com. Obtenido de https://www.gestiopolis.com/ administracion-empresarial-y-economiavenezolana/

Solís Sierra, M. I. (2018). Desarrollo de un modelo de gestión administrativa para el sistema de vinculación con la comunidad del Instituto Tecnológico Superior Juan Bautista Aguirre del Cantón Daule, Zona 5-Guayas. Universidad y Sociedad, 10.

Valdés, D. R. (02 de 12 de 2019). scielo. Obtenido de h t t p: / / s c i e l o.s ld.cu / scielo.php?script $=$ sci_arttext\&pid $=\$ 2310-340$ X2019000300392\&lang=es

Valero, J. (09 de 2016). El proceso administrativo en las MiPyMEs. Clamati Salidaria, 22-24. Obtenido de http://tlamati.uagro.mx/t7e1/40.pdf 